Ambiente \& Água - An Interdisciplinary Journal of Applied Science
ISSN 1980-993X - doi:10.4136/1980-993X
www.ambi-agua.net
E-mail: ambi-agua@agro.unitau.br

\title{
Determinação simultânea de estriol, $\beta$-estradiol, $17 \alpha$-etinilestradiol e estrona empregando-se extração em fase sólida (SPE) e cromatografia líquida de alta eficiência (HPLC)
}

\author{
doi: $10.4136 / a m b i-a g u a .1346$ \\ Received: 19 Mar. 2014; Accepted: 17 Sep. 2014 \\ Marina da Silva Daniel; Elizabete Campos de Lima* \\ Universidade Federal do ABC (UFABC), Santo André, SP, Brasil \\ *Autor correspondente: e-mail: elizabete.lima@ufabc.edu.br, \\ marina.daniel.91@gmail.com
}

\section{RESUMO}

Os hormônios $\beta$-Estradiol, estrona, estriol e $17 \alpha$-etinilestradiol vêm sendo detectados em efluentes principalmente porque os atuais processos de tratamento de esgotos são incapazes de removê-los, sendo uma parte descartada em corpos receptores. Estas substâncias causam danos à saúde humana e ambientais (organismos aquáticos e terrestres) por isso o seu monitoramento é necessário. No presente trabalho, empregou-se extração em fase sólida (SPE) utilizando cartuchos adsorventes de octadecil sílica $\left(\mathrm{C}_{18}\right)$ e análise por cromatografia líquida de alta eficiência (HPLC) para a determinação simultânea desses hormônios em amostra de água do Córrego Tubarão. A análise cromatográfica foi realizada em modo isocrático, fase móvel $50 \% \quad \mathrm{ACN}: \mathrm{H}_{2} \mathrm{O} \mathrm{pH} 3,0$; coluna $\mathrm{C}_{18}$, vazão $1 \mathrm{mLmin}^{-1}$, injeção $10 \mu \mathrm{L}, \lambda=281 \mathrm{~nm}, \mathrm{~T}=40^{\circ} \mathrm{C}$. Os limites de quantificação encontrados foram de $3,75 \mu \mathrm{gL}^{-1}$ para o $17 \alpha$-etinilestradiol a $7,75 \mu \mathrm{gL}^{-1}$ para o estriol. Os limites de detecção encontrados foram de $1,12 \mu \mathrm{gL}^{-1}$ para o $17 \alpha$-etinilestradiol a $2,32 \mu \mathrm{gL}^{-1}$ para o estriol. Na faixa de 0 a 60 $\mu \mathrm{gL} \mathrm{L}^{-1}$, os hormônios analisados apresentaram altos coeficientes de correlação $\left(\mathrm{R}^{2} \geq 0,99\right)$. A precisão (inter e intra-ensaio) avaliada em 4 diferentes níveis de concentração apresentou coeficiente de variância (\%CV) e exatidão inferiores a 3\%. Foram realizados diferentes testes de extração SPE mostrando que o condicionamento utilizando-se acetonitrila, eluição com metanol mostrou valores de recuperação superiores a 75\%. A metodologia validada foi aplicada na análise de uma amostra de água do córrego Tubarão (Santo André, SP) destacando-se a presença dos hormônios estriol $0,4 \mathrm{gL}^{-1}$ e estrona $0,32 \mathrm{gL}^{-1}$. A metodologia proposta apresentou-se adequada para a determinação de hormônios estrógenos em águas naturais.

Palavras-chave: contaminação, desreguladores endócrinos, poluentes emergentes, poluição aquática.

\section{Simultaneous determination of estriol, $\beta$-estradiol, $17 \alpha$-ethinyl estradiol and estrone employing solid phase extraction (SPE) and high performance liquid chromatography (HPLC)}

\section{ABSTRACT}

The hormones $\beta$-estradiol, estrone, estriol and $17 \alpha$-ethinyl estradiol have been detected in effluents because the current processes of sewage treatment are unable to remove them; the 
residue is being discarded into receiving bodies. These substances cause harm to human and environmental health (aquatic and terrestrial organisms), which is why there is a need for monitoring them. In the present work, we used SPE methodology using C18 cartridges and a HPLC method for simultaneous determination of these hormones in effluent samples. HPLC analysis was performed in isocratic mode, mobile phase $50 \% \mathrm{ACN}: \mathrm{H}_{2} \mathrm{O} \mathrm{pH} 3.0, \mathrm{C} 18$ column, flow $1 \mathrm{mLmin}^{-1}$ Injection $10 \mu \mathrm{L}, \lambda=281 \mathrm{~nm}, \mathrm{~T}=40^{\circ} \mathrm{C}$. The measurements ranged from $3.75 \mu \mathrm{gL}^{-1}$ for $17 \alpha$-ethinylestradiol to $7.75 \mu \mathrm{gL}^{-1}$ for estriol. Detection limits varied from $1.12 \mu \mathrm{gL}^{-1}$ for $17 \alpha$-ethinylestradiol to $2.32 \mu \mathrm{gL}^{-1}$ for estriol. In the range of $0-60 \mu \mathrm{gL}^{-1}$ the hormones analyzed showed high correlation coefficient $\left(\mathrm{R}^{2} \geq 0.99\right)$. The precision (inter- and intra-assay) assessed at 4 different concentration levels presented coefficient of variance (\%CV) and accuracy lower than 3\%. Different tests have been performed showing that the SPE extraction using acetonitrile elution with methanol showed recovery values higher than 75\%. The validated methodology was applied in the analysis of a sample of water from Tubarão Stream (Santo André, SP) highlighting the presence of the hormones estriol $0.4 \mu \mathrm{gL}^{1}$ and estrone $0.32 \mu \mathrm{gL}^{-1}$. The proposed methodology was found to be adequate for the determination of estrogen hormones in natural water.

Keywords: contamination, emerging pollutants, endocrine disrupters, water pollution.

\section{INTRODUÇÃO}

Desreguladores endócrinos são substâncias exógenas capazes de causar efeitos adversos à saúde, interferindo no sistema endócrino de organismos aquáticos, e nos últimos anos têm sido um dos principais tópicos de pesquisa em diferentes áreas do conhecimento (Chang et al., 2009).

$\mathrm{O}$ estrogênio sintético $17 \alpha$-etinilestradiol (EE2), amplamente usado na medicina em terapias de reposição e métodos contraceptivos, e os naturais estrona (E1), 17 $\beta$-estradiol (E2) e estriol (E3) são considerados como responsáveis pela maioria dos efeitos de desregulação endócrina (Reis Filho et al., 2006) pois afetam o sistema endócrino de peixes em concentrações de $1 \mathrm{ngL}^{-1}$, podendo ser encontrados no ambiente em concentrações da ordem de $\mu g L^{-1}$ a ngL $L^{-1}$ (Bila e Dezotti, 2007).

A persistência da atividade dos estrogênios tem origem na contínua introdução em ambientes aquáticos por meio da disposição inadequada de esgoto sanitário e industrial, como também pelo uso de lodo ativado de estação de tratamento de esgoto na agricultura. (Reis Filho et al., 2006; Qiujin et al., 2009; Lin et al., 2013).

O uso de águas superficiais para o consumo humano, bem como a transformação de seus mananciais como receptores de esgotos sanitários tratados ou não, tem sido os principais motivos de preocupação acerca da contaminação das fontes de água.

Há trabalhos que demonstraram que as substâncias estrogênicas não só são importantes na fase aquosa, mas também podem se acumular em sedimentos marinhos e assim afetar os organismos presentes no meio. Porém, pouco é conhecido sobre a exposição de organismos em ambientes aquáticos a substâncias estrogênicas presentes em sedimentos marinhos (Legler et al., 2002).

Diante da problemática de poluição dos compartimentos aquáticos que servem de fonte de captação de água para o abastecimento público, há consideráveis possibilidades de contaminação também da água potável, por esses estrógenos. Além disso, os tratamentos convencionais de água e de esgoto sanitário não são capazes de remover completamente tais substâncias, que permanecem no meio aquático. Diversos trabalhos têm sido dedicados a desenvolver métodos para determinação e quantificação de desreguladores endócrinos e avaliação de sua atividade estrogênica em organismos aquáticos (Chang et al., 2009). 
Técnicas de preparo de amostras (SPE, SPME) (Girotto et al., 2007; Servos et al., 2005) e cromatográficas (LC, GC) com diferentes detectores (DAD, FLD, MS) e outras fundamentadas em testes toxicológicos (YES, ELISA, E-screen, ELRA) (Verbinenn et al., 2010) têm sido desenvolvidas.

Estudos conduzidos por pesquisadores de Campinas, SP, apontaram a presença de hormônios estrógenos no Rio Atibaia, que abastece 92\% do município (Ghiselli, 2006). Em 2006, Sodré e colaboradores (Sodré et al., 2010) identificaram, neste mesmo ecossistema aquático, em um ponto à jusante da cidade de Campinas, níveis de 17 $\beta$-estradiol e $17 \alpha$-etinilestradiol que chegaram a 2,51 e $0,31 \mu \mathrm{g} \mathrm{L}{ }^{-1}$, respectivamente.

No presente trabalho foi desenvolvida uma metodologia de extração e clean-up utilizando cartuchos de extração em fase sólida (SPE) comerciais $\left(\mathrm{C}_{18}\right)$ e também uma metodologia via cromatografia líquida de alta eficiência (HPLC) para a determinação simultânea de estriol, $\beta$-estradiol, $17 \alpha$-etinilestradiol e estrona em amostra de agua do córrego Tubarão na divisa entre Santo André e Mauá que deságua na Represa Billings.

\section{MATERIAIS E MÉTODOS}

Desenvolveu-se e otimizou-se uma metodologia HPLC com detecção UV utilizando-se um sistema Agilent 1220 Infinity gradiente equipado com detector DAD, sistema de degaseificação online, forno para coluna, injetor automático e software de aquisição e análise de dados OpenLab EZChrom. As condições de análise foram: fase móvel 50\% ACN: $\mathrm{H}_{2} \mathrm{O}$ acidificada com uma solução $3 \mathrm{mmolL}^{-1}$ de ácido fosfórico (v/v) pH 3,0; coluna $\mathrm{C}_{18}(2,1 \mathrm{~mm}$ x $250 \mathrm{~mm}, 5 \mu \mathrm{m}$ procedência Supelco), fluxo $1 \mathrm{mLmin}^{-1}$, injeção $10 \mu \mathrm{L}, \lambda=281 \mathrm{~nm}$, $\mathrm{T}=40^{\circ} \mathrm{C}$. Para a otimização do método foram utilizados os padrões de hormônios estriol, $\beta$-estradiol, 17 $\alpha$-etinilestradiol e estrona (procedência Sigma-Aldrich). Os padrões de estrogênio, grau analítico foram pesados em balança analítica e dissolvidos com os solventes da fase móvel antes do uso na concentração de $100 \mathrm{mgL}^{-1}$ e armazenados em frasco âmbar com $2 \mathrm{~mL}$ de capacidade e conservados em geladeira antes do uso por até 48horas. A metodologia foi validada segundo o protocolo ICHQ2R1 (ICH, 2005).

No desenvolvimento da metodologia de extração em fase sólida foram utilizados cartuchos SPE C18 (Strata Phenomenex C18-E, 100 mg, 3 mL).

\section{RESULTADOS E DISCUSSÃO}

Na Tabela 1 são mostrados os limites de detecção e quantificação obtidos pela metodologia HPLC desenvolvida e otimizada. Os limites de detecção e quantificação foram avaliados utilizando-se curva analítica empregando-se a leitura do branco analítico do sistema. A curva analítica foi obtida a partir da análise cromatográfica de diluições em água a partir de uma solução estoque contendo os 4 padrões de estrogênio de $100 \mathrm{mgL}^{-1}$.

Tabela 1. Limites de detecção (LD), quantificação (LQ) obtidos para os hormônios analisados e linearidade na faixa de concentração estudada $\left(0\right.$ a $\left.60 \mu \mathrm{gL}^{-1}\right)$.

\begin{tabular}{lccc}
\hline Hormônio & $\mathbf{L Q}\left(\boldsymbol{\mu \mathbf { g L } ^ { - \mathbf { 1 } } )}\right.$ & $\mathbf{L D}\left(\boldsymbol{\mu \mathbf { g L }} \mathbf{- 1}^{\mathbf{1}}\right)$ & $\begin{array}{c}\text { Linearidade obtida e coeficiente de correlação } \\
\text { obtido }\left(\mathbf{R}^{\mathbf{2}}\right)\end{array}$ \\
\hline estriol & 7,75 & 2,32 & $\mathrm{y}=7397 \mathrm{x}+11,579, \mathrm{R}^{2}=0,9998$ \\
$\beta$-estradiol & 5,41 & 1,62 & $\mathrm{y}=10624 \mathrm{x}+150,75, \mathrm{R}^{2}=0,9999$ \\
$17 \alpha$-etinilestradiol & 3,75 & 1,12 & $\mathrm{y}=9784 \mathrm{x}+231,23, \mathrm{R}^{2}=0,9998$ \\
estrona & 5,25 & 1,57 & $\mathrm{y}=6956 \mathrm{x}+551,82, \mathrm{R}^{2}=0,9999$ \\
\hline
\end{tabular}


Na Figura 1 é mostrada a separação otimizada obtida experimentalmente.

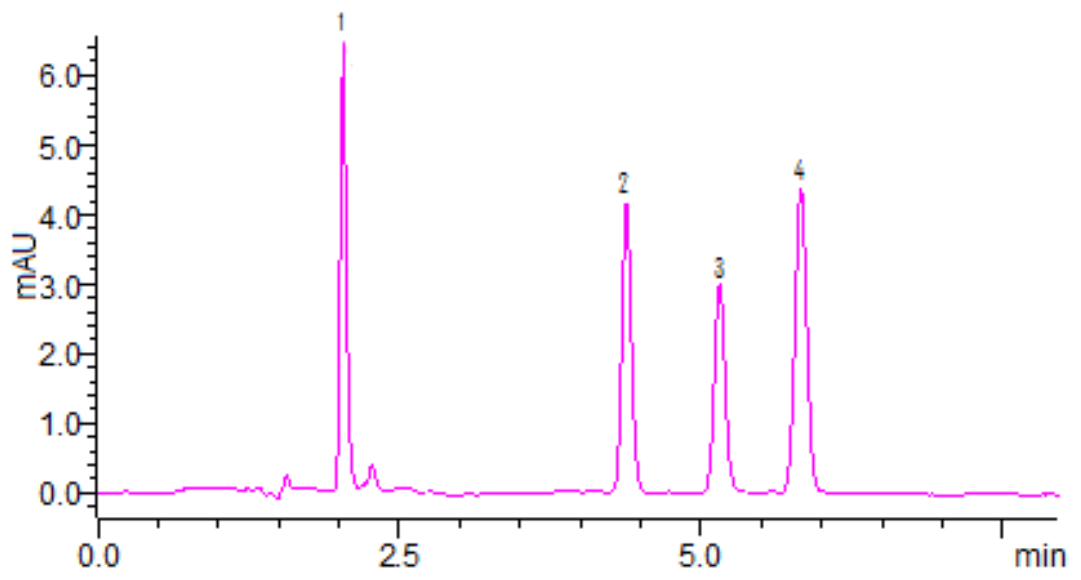

Figura 1. Cromatograma obtido para (1) estriol, (2) $\beta$-estradiol, (3) $17 \alpha$-etinilestradiol e (4) estrona. As condições de análise foram: fase móvel $50 \%$ ACN: $\mathrm{H}_{2} \mathrm{O}$ acidificada com solução $3 \mathrm{mmolL}^{-1} \mathrm{de}$ $\mathrm{H}_{3} \mathrm{PO}_{4} \mathrm{pH} 3,0(\mathrm{v} / \mathrm{v})$ coluna $\mathrm{C}_{18}$, fluxo $1 \mathrm{mLmin}^{-1}$, injeção $10 \mu \mathrm{L}$, $\lambda=281 \mathrm{~nm}, \theta=40^{\circ} \mathrm{C}$.

Observando-se a Tabela 1 e a Figura 1 verifica-se que a separação cromatográfica obtida é excelente com resolução na linha de base para todos os analitos e com tempo de análise inferior a $7 \mathrm{~min}$. A metodologia proposta apresenta limites de detecção e quantificação adequados, e os valores obtidos são satisfatórios para a determinação de hormônios estrógenos em esgoto doméstico e água tratada. A validação foi realizada utilizando-se replicata de amostra e de injeção $(n=36$, sendo 3 replicatas de cada concentração e 3 injeções diferentes de cada uma das 3 replicatas de amostras).

Na Tabela 2 são apresentados os valores obtidos para as figuras de mérito avaliadas.

Tabela 2. Valores de coeficiente de variância $(\% \mathrm{CV})$ para as figuras de mérito avaliadas na validação da metodologia HPLC utilizando o protocolo ICHQ2R1 ( $\mathrm{n}=36)$.

\begin{tabular}{|c|c|c|c|c|c|c|c|c|c|c|c|c|}
\hline \multirow{3}{*}{ Compostos } & \multirow{2}{*}{\multicolumn{4}{|c|}{$\begin{array}{l}\text { intra-ensaio }(\% \mathrm{CV}) \\
\begin{array}{l}\text { Níveis de concentração } \\
\text { avaliados }\left(\mu \mathrm{gL}^{-1}\right)\end{array}\end{array}$}} & \multirow{2}{*}{\multicolumn{4}{|c|}{$\begin{array}{l}\text { inter-ensaio }(\% \mathrm{CV}) \\
\begin{array}{l}\text { Níveis de concentração } \\
\text { avaliados }\left(\mu \mathrm{gL}^{-1}\right)\end{array}\end{array}$}} & \multirow{2}{*}{\multicolumn{4}{|c|}{$\begin{array}{l}\text { Exatidão }(\% \mathrm{CV}) \\
\begin{array}{l}\text { Níveis de concentração } \\
\text { avaliados }\left(\mu \mathrm{gL}^{-1}\right)\end{array}\end{array}$}} \\
\hline & & & & & & & & & & & & \\
\hline & 1,25 & 2,5 & 5 & 10 & 1,25 & 2,5 & 5 & 10 & 1,25 & 2,5 & 5 & 10 \\
\hline estriol & 0,1 & 3,2 & 1,9 & 1 & 0,1 & 3,2 & 1,9 & 1 & 0,1 & 2,6 & 1 & 0,2 \\
\hline$\beta$-estradiol & 2,2 & 1,4 & 0,3 & 2,4 & 1,7 & 0,8 & 0,2 & 0,1 & 1,62 & 1 & 0,2 & 0,2 \\
\hline $17 \alpha-$ etinilestradiol & 0,5 & 2,4 & 0,9 & 0,4 & 0,3 & 2,6 & 1 & 0,3 & 2 & 0,8 & 0,5 & 0,3 \\
\hline estrona & 2,9 & 0,1 & 1,5 & 0,2 & 2 & 0,2 & 0,8 & 0,2 & 2,1 & 1 & 0,2 & 0,2 \\
\hline
\end{tabular}

A metodologia cromatográfica proposta apresenta valores de precisão e exatidão para os quatro níveis de concentração estudados aceitáveis e menores que 3\%. Os valores obtidos são satisfatórios para a determinação de hormônios estrógenos em esgoto doméstico, água tratada e cursos de água natural, uma vez que essas substâncias têm potencial presença em água potável, o que exige o seu monitoramento utilizando-se métodos analíticos que alcancem níveis de traços. 
Para o desenvolvimento do processo de extração e clean-up dos hormônios estudados foram utilizados $100 \mathrm{~mL}$ de uma solução aquosa contendo $1 \mathrm{mgL}^{-1}$ de cada um dos hormônios em análise. Baseado no trabalho de Verbinenn et al. de 2010 publicado na revista Química Nova. Foram realizados testes de condicionamento e eluição dos cartuchos C18 comercias conforme é mostrado no fluxograma da Figura 2:

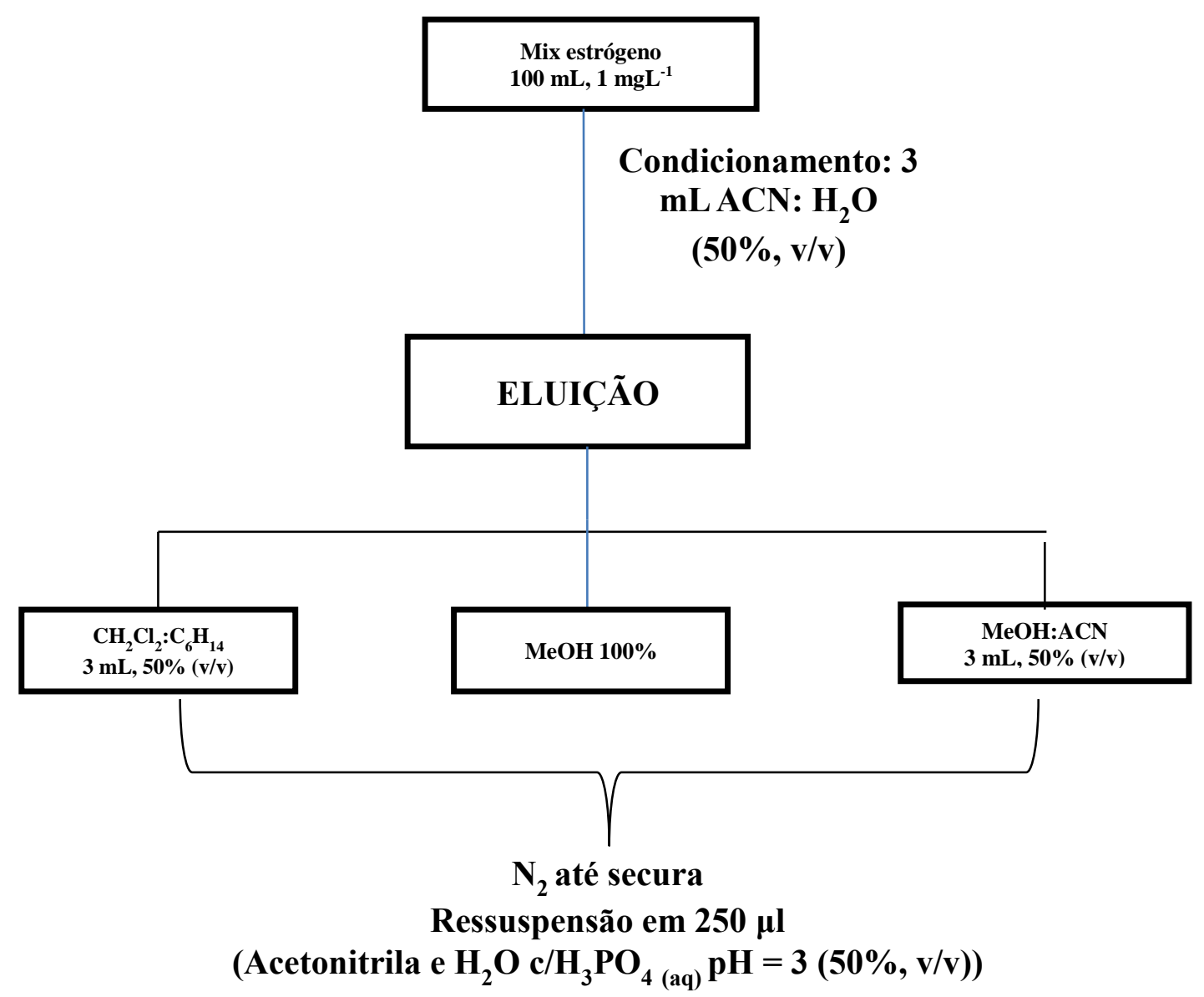

Figura 2. Esquema mostrando os testes experimentais realizados para extração e clean-up dos hormônios estrógenos utilizando cartuchos SPE C18.

A partir dos testes experimentais realizados e após a quantificação dos extratos SPE obtidos através da metodologia HPLC desenvolvida pode-se afirmar que o processo que gerou os melhores resultados, avaliados em termos de recuperação para os compostos analisados $(\% \mathrm{R})$ (Miller e Miller, 2005) foi a rota de extração utilizando o condicionamento do cartucho SPE com $3 \mathrm{~mL}$ de $\mathrm{ACN} ; \mathrm{H}_{2} \mathrm{O}$ (50\%, v/v) seguida da eluição em metanol (MEOH). Na Tabela 3 são mostrados os valores de recuperação alcançados pelo processo. Os valores são correspondentes à análise de triplicata de preparo de amostra seguida da triplicada de injeção de cada uma das amostras $(n=36)$. A recuperação dos analitos foi obtida comparando-se a concentração obtida após o ensaio e a adicionada inicialmente.

$\mathrm{Na}$ Tabela 3 observa-se que os valores obtidos variaram de 88,6 a $78,4 \%$ e são reprodutíveis com base nos valores de exatidão apresentados.

Foram realizados testes de sensibilidade utilizando-se o hormônio estanozolol e verificou-se que o mesmo não coelui com nenhum dos picos referentes aos hormônios estrógenos estudados assim como não provoca modificações no tempo de retenção dos mesmos. 
Tabela 3. Valores de Recuperação alcançados para a extração SPE com cartucho C18 utilizando-se condicionamento com acetonitrila:água $(50 \%, \mathrm{v} / \mathrm{v})$ seguida da eluição com metanol para os 4 hormônios estudados.

\begin{tabular}{lcc}
\hline \multicolumn{1}{c}{ Hormônios } & $\boldsymbol{\% R}$ & Exatidão $(\% \mathbf{C V})$ \\
\hline estriol & $88,6 \pm 0,8$ & 0,009 \\
$\beta$-estradiol & $86,6 \pm 0,4$ & 0,004 \\
$17 \alpha$-etinilestradiol & $78,4 \pm 0,8$ & 0,010 \\
estrona & $84 \pm 1,4$ & 0,017 \\
\hline
\end{tabular}

A metodologia validada foi aplicada a uma amostra de água retirada do córrego Tubarão que fica localizado na divisa entre os municípios de Santo André e Mauá (SP) e deságua na represa Billings. Na Figura 3 é mostrado o cromatograma para a amostra de água analisada. Foi submetida à análise uma alíquota de $100 \mathrm{~mL}$ da amostra coletada.

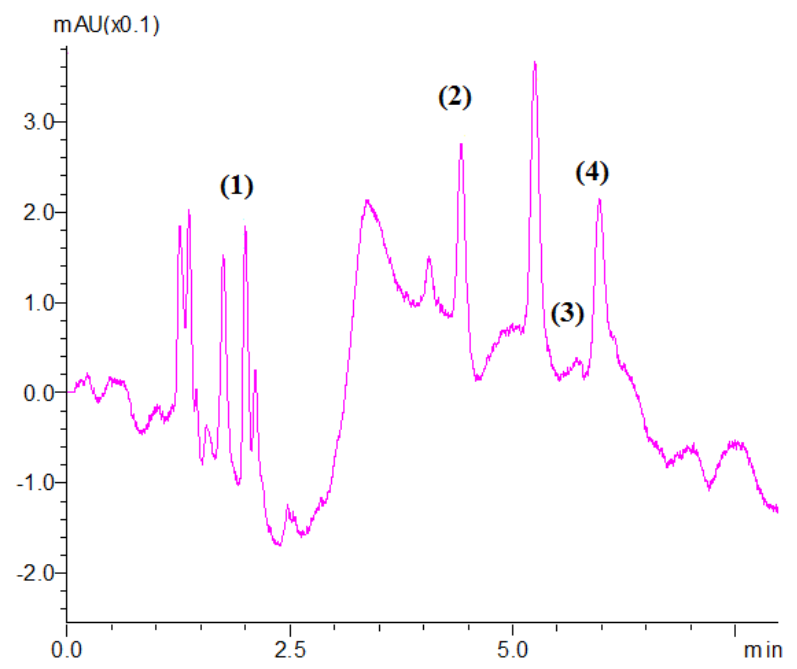

Figura 3. Cromatograma obtido para amostra de água do córrego Tubarão da região metropolitana de Santo André. As condições analíticas são as mesmas descritas na Figura 1.

A análise da amostra de água revelou a presença de estriol, $\beta$-estradiol, $17 \alpha$-etinilestradiol e estrona. A identificação dos picos foi feita através de pureza espectral e adição de padrão destaca-se a presença dos hormônios estriol $0,4 \mathrm{gL}^{-1}$ e estrona $0,32 \mathrm{gL}^{-1}$ na amostra analisada.

\section{CONCLUSÃO}

A metodologia proposta apresenta limites de detecção e quantificação adequados, além de valores de precisão e exatidão para os níveis de concentração estudados e menores que $3 \%$. Os valores obtidos são satisfatórios para a determinação de hormônios estrógenos em esgoto doméstico, água tratada e efluentes uma vez que essas substâncias têm potencial de presença em água potável, o que exige o seu monitoramento utilizando-se métodos analíticos que 
alcancem níveis de traços uma vez que os tratamentos atuais de água e esgoto não são capazes ainda de retirar esses compostos durante o processo.

\section{AGRADECIMENTOS}

Os autores agradecem a empresa Chemical Trends pela doação dos padrões de hormônios estrógenos utilizados para a realização do presente trabalho, ao Dr. Clovis Lucio da Silva na etapa de desenvolvimento da metodologia cromatográfica e a FAPESP, Processo 2013/12569-8 pelo apoio financeiro.

\section{REFERÊNCIAS}

BILA, D. M.; DEZOTTI, M. Desreguladores endócrinos no meio ambiente: efeitos e conseqüências. Quim. Nova, v. 30, p. 651-666, 2007. http://dx.doi.org/10.1590/S010040422007000300027

CHANG, H.S.; CHOO, K. H.; CHO, S.J. The methods of identification, analysis, and removal of endocrine desrupting compounds (EDCs). J. Hazard. Mater., v. 172, p. 1-12, 2009. http://dx.doi.org/10.1016/j.jhazmat.2009.06.135

GHISELLI, G. Avaliação da qualidade das águas destinadas ao abastecimento público na região de Campinas: ocorrência e determinação de Interferentes Endócrinos (IE) em produtos farmacêuticos e de higiene pessoal 2003-2006. 2006. 130f. Dissertação (Doutorado em Ciências área de concentração: Química) - Instituto de Química da Unicamp, Campinas, 2006.

GIROTTO, G.; NOGUEIRA, K. L.; MOURA, J. A.; SOUZA, J. B.; GIACOMETTI, L. L.; MARCHI, M. R. R. et al. Estrógenos em água: otimização da extração em fase sólida utilizando ferramentas quimiométricas. Ecl. Quím., v. 32, n. 2, p. 61-67, 2007. http://dx.doi.org/10.1590/S0100-46702007000200010

INTERNATIONAL CONFERENCE ON HARMONISATION OF TECHNICAL REQUIREMENTS FOR REGISTRATION OF PHARMACEUTICALS FOR HUMAN USE (ICH). Harmonised tripartite guideline - validation of analytical procedures: text and methodology Q2(R1). Geneva, 2005.

QIUJIN, Z.; LIPING, W.; SHENGFANG, W.; WASSWA, J.; XIAOHONG, G.; JIAN, T. Selectivity of molecularly imprinted solid phase extraction for sterol compounds. Food Chem., v. 113, n. 2, p. 608-615, 2009. http://dx.doi.org/10.1016/j.foodchem.2008.07.044.

LEGLER, J.; DENNEKAMP, M.; VETHAAK, A. D.; BROWWER, A.; KOEMAN, J. H.; VAN DER BURG, B. et al. Detection of estrogenic activity in sediment-associated compounds using in vitro reporter gene assays. Sci. Total Environ., v. 293, p.69-83, 2002. http://dx.doi.org/10.1021/jf052864f

LIN, Z.; HE, Q.; WANG, L.; WANG, X.; DONG, Q.; HUANG, C. Preparation of magnetic multi-functional molecularly imprinted polymer beads for determining environmental estrogens in water samples. J. of Haz. Mat., v. 252- 253, p. 57- 63, 2013. http://dx.doi.org/10.1016/j.jhazmat.2013.02.041

MILLER, J. N.; MILLER, J. C. Statistics and chemometrics for analytical chemistry. 5th Edition. London: Pearson Prentice Hall, 2005. 268 p. 
REIS FILHO, R. W.; ARAUJO, J. C.; VIEIRA, E. M. Hormônios sexuais estrógenos: contaminantes bioativos. Quim. Nova, v. 29, p. 817-822, 2006. http://dx.doi.org/10.1590/S0100-40422006000400032

SERVOS, R. M.; BENNIE, T. D.; BURNISON, K. B.; JURKOVIC, A; MCINNIS, R.; NEHELI, T. et al. Distribution of estrogens, 17h-estradiol and estrone in Canadian municipal wastewater treatment plants. Science of Tot. Environ., v. 336, n. 1, p. 155 170, 2005. http://dx.doi.org/10.1016/j.scitotenv.2004.05.025

SODRÉ, F. F.; MONTAGNER, C. C.; LOCATELLI, M. A. F.; JARDIM, W. F. Sistema limpo em linha para extração em fase sólida de contaminantes emergentes em águas naturais. Quim. Nova, v. 33, p. 216-219, 2010. http://dx.doi.org/10.1590/S010040422010000100037

VERBINENN, R. T.; NUNES, G. S.; VIEIRA, E. M. Determinação de hormônios estrógenos em água potável utilizando CLAE-DAD. Quim. Nova, v. 33, p. 1837-1842, 2010. http://dx.doi.org/10.1590/S0100-40422010000900003 